Research Article

\title{
Modelling and Simulation of Knee simulator testing Machine using Lab view
}

\author{
R.B.Durairaj ${ }^{\dot{A}^{*}}$, M.Sivasankar $^{\dot{B}}$ and A.Krishnamoorthy ${ }^{\dot{A}}$ \\ ${ }^{\dot{A}}$ Faculty of Mechanical Engineering, Sathyabama University, Chennai. \\ ${ }^{\dot{B}}$ Department of Mechanical Engineering, SKP Engineering College, Tiruvannamalai.
}

Accepted 10 January 2014, Available online 01 February 2014, Special Issue-2, (February 2014)

\begin{abstract}
In these recent trends, over $70 \%$ of osteosarcoma affects large bones in the lower extremity (femur and tibia). Most of the cases are reported for children below 20 years, and require surgery involving prosthetic reconstruction. so, the development of Artificial Prosthesis is the Prominent Solution for this great Cause. Osteosarcoma is an aggressive malignant neoplasm arising from primitive transformed cells of mesenchymal origin that exhibit osteoblastic differentiation and produce malignant osteoid. It is the most common histological form of primary bone cancer. Before implant of the Prosthesis it should be tested nearly 10 million cycles to withstand 10 years of prosthetic life in the human body. Knee Simulator is the Testing Machine which helps in testing of this artificial Prosthesis by achieving various motions of the knee joint such as Flexion, Extension, Tibial Rotation, etc by applying the Artificial load for the joint constantly. In this Paper the Flexion - Extension motion of the Knee joint has been tested from 0 to $60^{\circ}$ with the constant load. this simulator has been designed and developed in such a way that it can withstand the loads and vibrations during testing. And this hardware is engaged with the computer control using the LABVIEW Software
\end{abstract}

Keywords: Knee simulator, knee joint, degrees of freedom.

\section{Introduction}

The knee joint is rendered with six degrees of freedom and Long characterized a functionally as a hinge joint, the true motion in this joint complex is much more intricate. As with most biological hinge joints, the knee motion consists of combined gliding and rolling actions of the distal end of the femur with respect to the proximal articular surface of the tibia, as well as the sliding motion of the patella on the femur Four ligaments are present in the knee joint, the medical collateral ligament, lateral collateral ligament, anterior cruciate ligament, and posterior cruciate ligament. In 1994, the Orthopaedic Biomechanics Laboratory at the Hennepin County Medical Centre in Minneapolis, Minnesota was developed the machine first made its public literature debut with the publication of "Dynamic Simulation Machine for Measurement of Knee Mechanics and Intra-Articular Pressures. Like most knee simulators, the EnduraTec machine was designed and built with the expressed purpose of measuring the dynamic motions, pressure loads and surface areas of the knee joint. In 1994 Johns Hopkins/ MTS knee simulator was developed with the joint project of Johns Hopkins University Orthopaedic Biomechanics Laboratory, MTS Systems, and the Mayo Clinic. The stated goal of the creation of the knee simulator was the experimentation on altered and unaltered cadaveric specimens to determine the effect of orthopaedic deficiencies through the surgical creation of

*Corresponding author: R.B.Durairaj

DOI: http://dx.doi.org/10.14741/ijcet/spl.2.2014.126 those deficiencies. With these literatures we have undergone various considerations and developed the simulator for the testing of artificial knee joint.

\section{Knee prosthesis}

This prosthesis is made up of Bio materials such as Ti 64 and UHMWPE (ultra high molecular weight polyethylene) and SS $17 \quad-4 \mathrm{PH}$. Ultra-high-molecular-weight polyethylene (UHMWPE) is a subset of the thermoplastic polyethylene also known as high-modulus polyethylene, (HMPE), or high-performance polyethylene (HPPE).

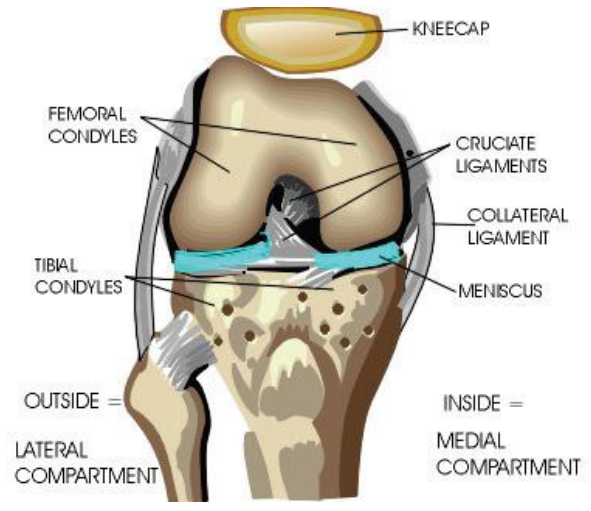

Fig. 1 Basic anatomy of human knee

It has extremely long chains, with a molecular weight usually between 2 and 6 million. The longer chain serves 666 | International Conference on Advances in Mechanical Sciences 2014 
to transfer load more effectively to the polymer backbone by strengthening intermolecular interactions. These results in a very tough material, with the highest impact strength of any thermoplastic presently made. EOS Titanium Ti64 is a pre-alloyed Ti6AlV4 alloy in fine powder form.

This well-known light alloy is characterized by having excellent mechanical properties and corrosion resistance combined with low specific weight and biocompatibility. This material is ideal for many high-performance engineering applications, for example in aerospace and motor racing, and also for the production of biomedical implants. Parts built in EOS Titanium Ti64 fulfil the requirements of ASTM F1472 regarding maximum concentration of impurities. the major applications of this $\mathrm{Ti} 64$ are direct manufacture of functional prototypes small series products, individualised products or spare parts requiring a combination of high mechanical properties and low specific weight. The knee joint 3D model shown in the fig has been converted in to the Rapid prototyping System format (STL File) in CATIA V5 and undergone the RPT Development.

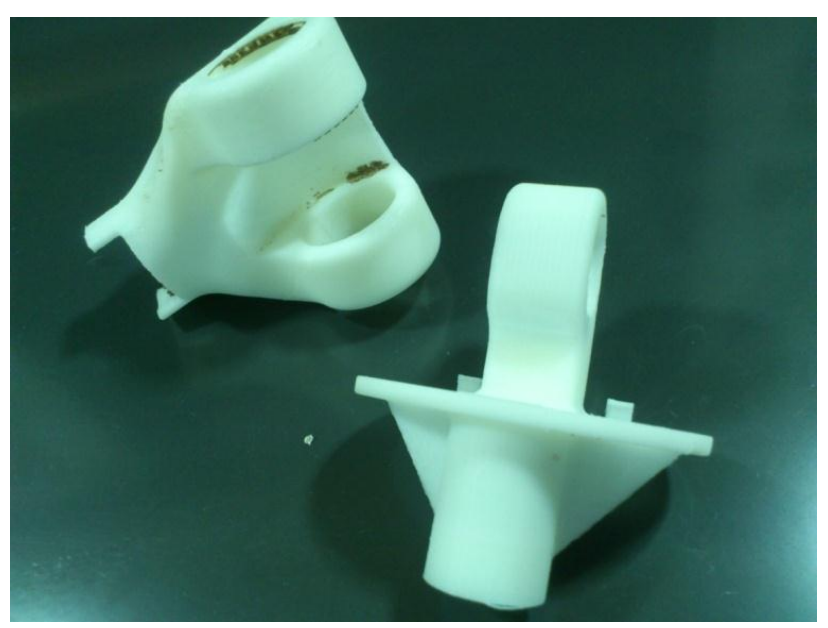

Fig. 2 Rapid prototyping model of knee prosthesis

\section{Knee simulator}

Both the modelling and development of prototype has been shown in this discussion on the knee simulator testing machine. This includes the various components of the knee simulator such as base structure, shaft assembly, motors, sensors etc. The brief about this component are discussed below.

\section{Base assembly}

The base assembly consist of Very Rigid structure as shown in the figure consist of both the model and manufactured component. This base frame consist of four base side bars with the dimension of 500 X 50 X 50 MM which is the thickness of $5 \mathrm{~mm}$ on four sides attached to the base plate is the rigid rectangle plate at the length of $450 \times 300 \mathrm{~mm}$ with the thickness of $20 \mathrm{~mm}$ as shown in figure. There is two bars at the top named Top Front bars which is at the Size of $300 \times 50 \times 50 \mathrm{MM}$ with $5 \mathrm{~mm}$ pipe thickness and the Side bars are at the dimension of $350 \mathrm{X}$
50X 50MM dimensions. The foot rest has been made with the cushioning bush for the effect of vibration Reduction at the bottom of the corner vertical channels.

\section{Shaft Assembly}

The Shaft of the Servo Motor and the Condoyle Centre Shaft have to align in the straight line for achieving maximum efficiency. This has been achieved with the Proper Dimensions of the simulator. this shaft assembly consist of Top slider, Motor Shaft holder, Bearings , Coupler, etc. From the Fig 3 it is showing about the Sub Assembly for the Shaft. From the left it is showing about the motor shaft, followed by the coupler and the coupler shaft which is used to transmit the power from the motor to the slider. Also this shaft is called Driver shaft and another shaft is called driven shaft which is used for holding the Top slider.

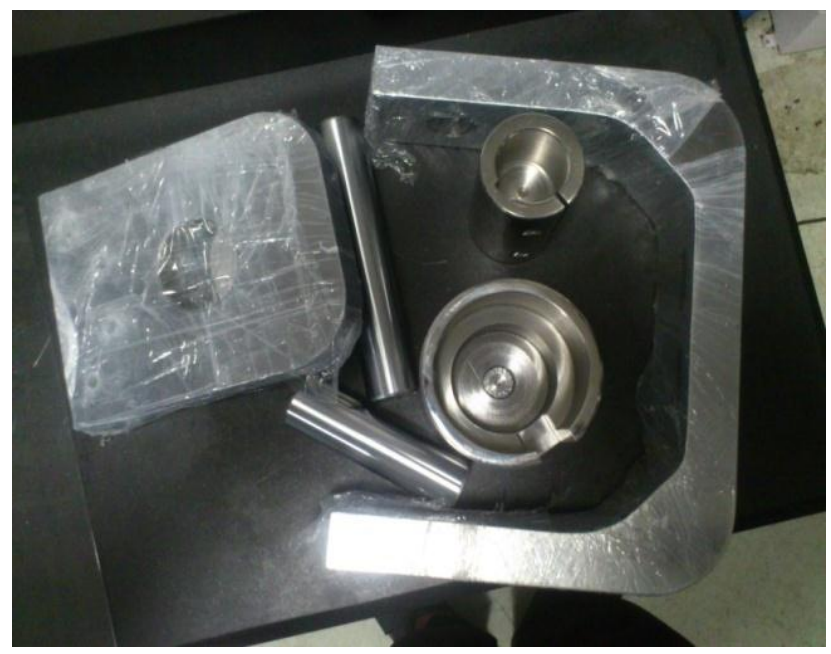

Fig. 3 Parts of the Shaft assembly

\section{Central Cylinder}

The central cylinder is the system which holds the Tibial tray of the Knee joint and also supplies the load for that. It has the Provision of load cell over the load cell seated which is connected to the pneumatic cylinder. In the top of the cylinder there will a pocketed surface for the provision of Tibial Tray. This cylinder has been made at the $\varnothing 93$ with the height of $120 \mathrm{~mm}$. In the down surface of the load cell seater the M18 thread is give for connecting with the Pneumatic Cylinder.

\section{Complete Assembly of the System}

The complete assembly of the system is shown in the Fig 4 It consist of the base assembly and the all the Sub Assembly such as Motor Sub assembly and Shaft Sub Assembly and mounted with the Knee joint over the Central Cylinder. This motor has been holder and clamped with the Base horizontal side bar for the Rigid support and for the Vibration Neutralization. The top of the Knee joint is connected to the Top Slider with the Help of the Femroal Extension Piece and some filler Piece and the 
Femoral part is placed at 5 to $10^{\circ}$ offset form the Central line as per the Standard of Testing.

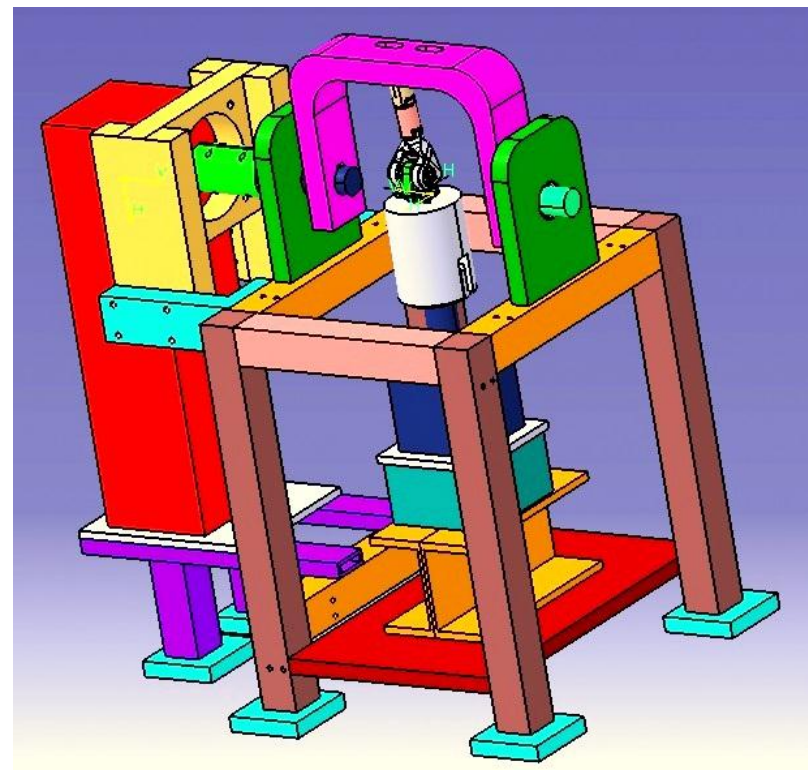

Fig. 4 Complete Assembly of the Knee Simulator

\section{Results and discussions}

The Entire Assembly of the KST 2 - V2 has been coupled with the Computer Control system with LABVIEW. This lab view system consists of a Feedback loop for supplying the Constant Load to the Knee joint. Fig 6.1 is showing the block diagram of the Feedback loop. From the above block diagram it is showing that the initial load has been given to the PID Controller and it sense the variation in the load and it will generates the signal to the computer control. This computer control sends the feed back to the Electro Pneumatic Convertor. This convertor actuates the pneumatic cylinder and makes the variation load in equilibrium.

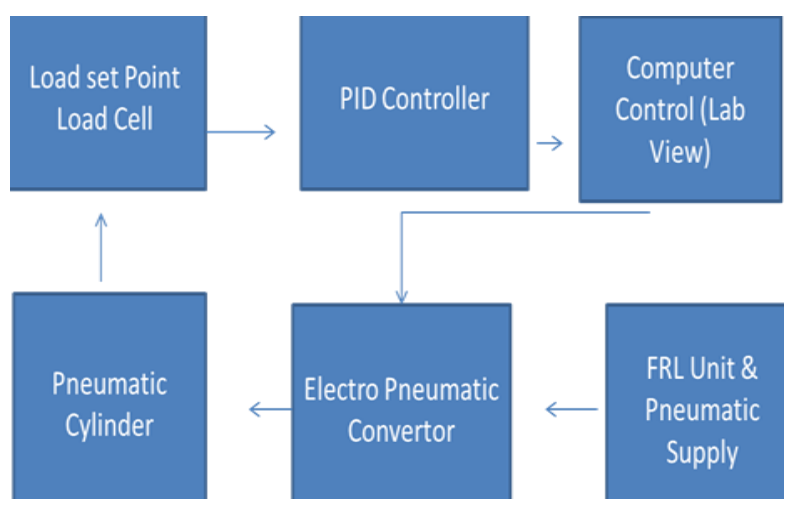

Fig. 5 block diagram of the knee simulator functioning

The lab view simulation consists of Two Windows such as Front Window and Back Window. In this Front window is mainly for showing the Outputs and the Back window for the Programming part. This lab view has all the Modules such as Wave From Graph, PID Controller, Numeric
Controller etc. With this help of this module the Cycle count ID, Run ID, Load Set Point, Current Load point has been described. The Wave Form graph has been connected to the Load Cell. This load cell variation has been displayed in this graph.

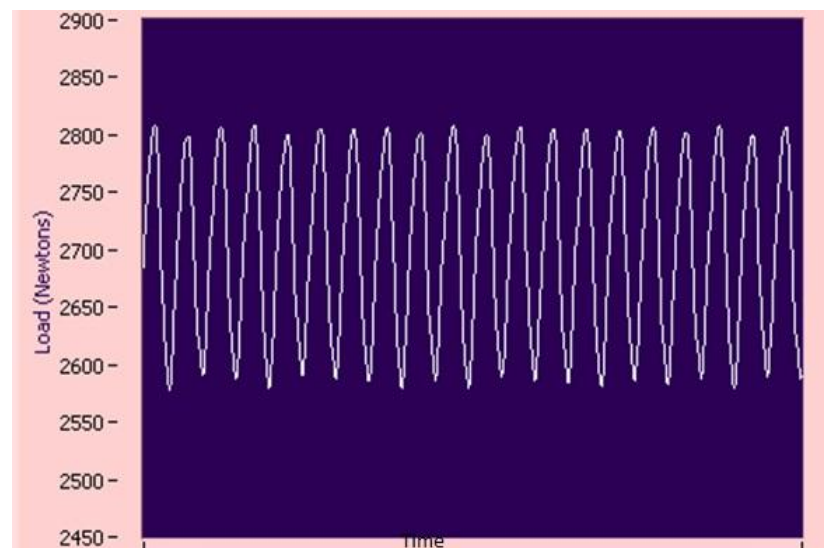

Fig. 6 Load Vs Time graph

The above figure 6 is showing about the relationship between the loads Vs Time. This wave from is the direct output of the load cell which constantly varies in their output values during the flexion motion of the knee joint. This load variation form $2600 \mathrm{~N}$ to $2800 \mathrm{~N}$ is the maximum variation of the load for the proper set point of $2700 \mathrm{~N}$.

\section{Conclusion}

Knee simulator testing machine has been developed and tested. The Flexion and Extension motion of the knee joint has achieved form 0 to $60^{\circ}$ with constant load. Also this hardware system has been successfully interfaced with LABVIEW with essential controller's modules. The variation in the set load due to variation of angle during flexion motion has been equalized with the help of the PID controller. In future this knee simulator testing machine will be developed with the additional features such as Tibial rotation motion along with the flexion motion for the knee joint. Also in this project we are not yet achieved the ISO standard gait cycle performance of testing because here we are applying the constant load of $2700 \mathrm{~N}$, but in case of the standard gait cycle this load will varying with respect to time. Also the acceleration of the servo motor for the flexion motion also varied for the different angular positions. So, this are the major cases has to take in consideration for the next simulator versions.

\section{References}

Ausbil dungs simulator, (1994). Diploma thesis, Darmstadt Technical University, Department of Computer Science Interactive Graphics Systems Group PP $452-460$.

Ahmed, A.M., Burke, D.L., Ducan, N.A., Chan, K.H., (1992). Ligament tension pattern in the \#exed knee in combined passive anterior translation and axial rotation. Journal of Orthopaedic Research 10, PP 854-867.

Audu, M.L. \& Davy, D.T. (1985). The influence of muscle model complexity in musculoskeletal motion modeling. Journal of Biomechanical Engineering, 107,PP 147-157 
Brand, R.A.; Crowninshield, (1982). R.D.; Wittstock, C.E.; Pederson, D.R.; Clark, C.R. \& Krieken, F.M. van A model of lower extremity muscular anatomy. Journal of Biomechanics, 104, PP 304-310

Blacharski, P. A.. Somerset, J. H.. Murray. D. G. (1975) A threedimensional study of the kinematics of the human knee. J. Biomechanics 8, PP 375-384.

DesJardins, J.D., Walker, P.S., Haider, H., Perry, J., (1999). An analysis of tibial component and soft tissue shear loads during a force controlled walking cycle for multiple TKR designs. Transactions of the Ninth Annual Meeting of the European Orthopaedic Research Society (EORS), June , Brussels, Belgium PP 345-350

DesJardins, J.D., Walker, P.S., Haider, H., Perry, J., (1999). The evaluation of TKR designs using a simulated walking cycle. Transactions of the Fourth Congress of the European Federation of National Assocoations of Orthopaedics and Traumatology (EFORT), June Brussels, Belgium. PP 450-46

Dijk. R. van. Huiskes. R. and Sclvik. G. (1979) Roentgensrereophologrammelric methods for the evaluation of Ihc three-dimensional kinematic behaviour and cruciate ligament length patterns of the human knee joint. J. Biomechunics 12,PP 727-731.

G.Fischer, Erstellungeiner (1994) 3D-Benutzerschnittstelle fiireinen medizinischen Ausbil dungs simulator,Diploma thesis, Darmstadt Technical University, Department of Computer Science Interactive Graphics Systems Group PP 556-56
H. Nunome, 1999 .Biomechanics of Kicking motion in soccer, Japanese Journal of Biomechanics in Sports and Exercise, Vol.3, No.2, PP.104- 110,

Y. Tanaka, N. Yamada, I. Masamori, 2004 and T. Tsuji, .Manipulability Analysis of lower Extremities Based on Human Joint-Torque Characteristics, Transaction of the Society of Instrument and Control Engineers, Vol.40, No.6, PP.612-61

Rosenberg, A.G., Verner, J.J., 1991. Galante, J.O., Clinical results of total knee revision using the total condylar III prosthesis. Clinical Orthopaedics and Related Research 273,PP 83-90.

Whiteside, L.A., Kasselt, M.R., Haynes, D.W., 1987. Varusvalgus and rotational stability in rotationally unconstrained total knee arthroplasty. Clinical Orthopaedics and Related Research 219, PP 147-157

William Roy Bar_eld, 2002 Donald T.Kirkendall, and Bing Yu, .Kinematic Instep Kicking Differences between Elite Female and Male Soccer Players,. Journal of Sports Science and Medicine, Vol. 1, No. 1, PP. 72-79

F. Schroder and P. RoBbach, (1994). "Managing the Complexity of Digital Terrain Models, Computers \& Graphics, Special Issue" on Modelling and Visualisation of Spatial Data in Geographic Information Systems. Pergamon Press, Oxford PP 234-241 